\title{
ESTUDO TEÓRICO DA DINÂMICA DA CONFLUÊNCIA BRASIL-MALVINAS
}

\author{
Cayo Prado Fernandes Francisco ${ }^{1}$ e IIson Carlos Almeida da Silveira ${ }^{2}$ \\ Recebido em 12 julho, 2004 / Aceito em 30 setembro, 2004 \\ Received July 12, 2004 / Accepted September 30, 2004
}

\begin{abstract}
The study of the mesoscale dynamics of the confluence of two western boundary currents was conducted in this work via a quasi-geostrophic inviscid $f$-plane two-layer ocean theoretical approach. The simplified vertical structure was calculated through the employment of a dynamic calibration scheme based on the dynamical mode structure of the Brazil-Malvinas Confluence Region. The Brazil-Malvinas mode structure was computed from climatological hydrography of the area delimited by $35^{\circ}-40^{\circ} \mathrm{S}$ and $50^{\circ}-60^{\circ} \mathrm{W}$. Two contour dynamics models were constructed: a linear and a nonlinear version. The basic flow configuration consisted of two converging western boundary currents that form a zonal eastward current in the upper layer. The lower layer flow was essentially divergent as a result of a westward zonal jet impinging on the western border. This vertical shear choice assured that the system was baroclinically unstable. The results of the model experiments showed that the presence of the confluence, the western meridional boundary and the barotropic mode in the dynamical structure of the basic flow favored long wave patterns. The three experiments conducted with the nonlinear model exhibited the development of both a reflection pattern and vortical dipoles. The dipoles pinched off from either the retroflection lobe (i.e., the primary crest of the wave train) or the primary trough when the baroclinically unstable current system was perturbed at the boundary vicinities. It was verified that the nonlinear model simulations followed the instability properties predicted by the linear model in terms of meander growth rates, phase speeds and most unstable wavelengths. This suggested that while the baroclinic instability mechanism was responsible for the temporal growth of the meanders, the nonlinear effects caused the dipole isolation and pinch off of the finite amplitude meanders. These dipoles could leave and propagate away from the current axis.
\end{abstract}

Keywords: Contour dynamics, calibrated layer models, baroclinic instability, Western Boundary Currents, Brazil-Malvinas Confluence.

RESUMO. 0 estudo da dinâmica de meso-escala da confluência de duas correntes de contorno oeste num oceano de duas camadas quase-geostrófico, não viscoso e inercial no plano $f$ foi conduzido neste trabalho. A estrutura vertical simplificada do modelo foi obtida através de calibração dinâmica em relação à estrutura modal da região da Confluência Brasil-Malvinas. Essa estrutura modal foi estimada a partir da climatologia da área compreendida entre $35^{\circ}-40^{\circ} \mathrm{S}$ e $50^{\circ}-60^{\circ} \mathrm{W}$. Dois modelos de dinâmica de contornos foram então desenvolvidos. Estes apresentaram sistema de correntes de contorno oeste com transporte simétrico e duas frentes de vorticidade, uma por camada. 0 escoamento na camada superior foi sempre convergente, formando um jato zonal que flui para leste. Já o escoamento na camada inferior foi divergente, com jato zonal de velocidades fluindo dominantemente para oeste. Tal configuração garantiu que o sistema fosse baroclinicamente instável. Os resultados dos experimentos mostraram que a presença da confluência, da fronteira meridional e do modo barotrópico no escoamento básico favoreceram ondas longas. Os três experimentos conduzidos com o modelo não-linear mostraram o desenvolvimento tanto do padrão de retroflexão como a emissão de vórtices e dipolos vorticais pelo lóbulo de retroflexão (crista primária na camada superior) e no cavado primário a partir de uma perturbação inicial imposta junto ao contorno oeste. Os cenários obtidos se assemelham qualitativamente àqueles observados na Confluência Brasil-Malvinas. Verificou-se também que, os resultados do modelo não linear seguem aqueles previstos pelo modelo linear em termos de taxas de crescimento, velocidades de fase e comprimento das ondas mais instáveis. Isto sugeriu que enquanto os processos de instabilidade baroclínica são responsáveis pelo crescimento temporal dos meandros, os efeitos não lineares causaram principalmente isolamento das estruturas vorticais em estruturas dipolares, que eventualmente se separam do eixo da corrente.

Palavras-chave: Dinâmica de contornos, calibração dinâmica, instablilidade baroclínica, Corrente de Contorno Oeste, Confluência Brasil-Malvinas.

\footnotetext{
${ }^{1}$ Instituto Oceanográfico da Universidade de São Paulo (I0-USP), Praça do Oceanográfico, 191 - Cidade Universitária - CEP: 05508-900, São Paulo, SP. Tel: (11) 3091-6578; Fax: (11) 3091-6610 - E-mail: cayoprado@ceres.io.usp.br

${ }^{2}$ Instituto Oceanográfico da Universidade de São Paulo (I0-USP), Praça do Oceanográfico, 191 - Cidade Universitária - CEP: 05508-900, São Paulo, SP. Tel: (11) 3091-6552 - E-mail: ilson@ipanema.ceres.io.usp.br ou ilson@ceres.io.usp.br.
} 


\section{INTRODUÇÃO}

Instabilidades em escoamentos geofísicos tais como meandramentos e ondas de vorticidade são feições comumente presentes em correntes de contorno oeste (CCOs) que se separam da costa. Em particular, aquelas que se separam em retroflexão, ou seja, a corrente se dobra sobre ela mesma exibindo um amplo meandro anticiclônico próximo ao contorno continental e meandros de sentidos opostos que se sucedem e decaem em amplitude em direção ao interior da bacia oceânica.

0 exemplo de retroflexão de CCO no Atlântico Sul é a separação da Corrente do Brasil (CB). Conforme Garzoli \& Garraffo (1989), a CB flui quase meridionalmente para sul até aproximadamente $36^{\circ} \mathrm{S}$ onde começa sua separação do continente estendendo-se até $38^{\circ} \mathrm{S}$. Esta separação ocorre devido à confluência das águas quentes da CB com as águas de origem subpolar da Corrente das Malvinas (CM). É talvez o caso mais evidente de convergência de duas correntes de contorno do oceano mundial, e está associado a intensa atividade vortical.

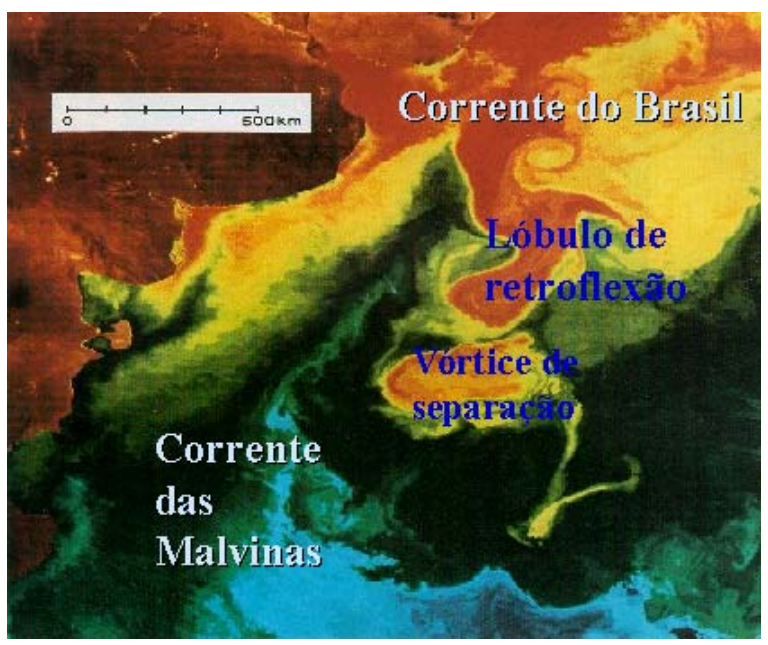

Figura 1 - Imagem AVHRR da confluência Brasil-Malvinas editada a partir de Olson et al. (1988).

Figure 1 - AVHR Image of the Brazil-Malvinas confluence edited from Olson et al. (1988).

Olson et al. (1988), valendo-se de observações de derivadores e imagens de satélites, estudaram a variabilidade de mesoescala da Confluência Brasil-Malvinas (Figura 1). Estes autores encontraram que os comprimentos de onda típicos dos meandros observados abrangiam 400 a 500 km e concluíram também que o lóbulo principal de retroflexão da CB frequentemente se alonga para sul e emite grandes grandes vórtices anticiclônicos dentro do giro subpolar. Formação de vórtices ciclônicos se desenvolvendo a partir de meandros ciclônicos a leste do lóbulo da retroflexão também são observados (Gordon \& Greengrove, 1986), assim como vórtices anticiclônicos que se desprendem da CB (Lentini et al., 2002). Investigações recentes de Goni \& Wainer (2002) através da utilização de dados altimétricos também indicam que as frentes da região de confluência CB-CM apresentam intensa variabilidade interanual com amplitudes médias anuais de $1^{\circ}$ a $6^{\circ}$ de latitude, que parecem estar fortemente relacionadas às flutuações do transporte baroclínico da CB.

Estudos anteriores sobre a dinâmica da separação de CCOs em retroflexão abordam o problema ou numericamente como Campos e Olson (1991), ou através de modelos analíticos estacionários como Ou \& De Ruijter (1986), Cessi (1991) e Nof \& Pichevin (1993). Embora esses trabalhos tenham respondido questões importantes a respeito da separação de CCOs, os modelos de simulações numéricas não permitem claramente o isolamento do mecanismo que atua na evolução do sistema dinâmico, enquanto os modelos analíticos estacionários ignoram o efeito da instabilidade geofísica no processo de crescimento dos meandros, e consequente formação de vórtices.

Mais recentemente, Silveira et al. (1999) aplicaram a técnica de dinâmica de contornos (DC) para um oceano semi-infinito inercial de $1 \frac{1}{2}$-camadas para investigar o processo de formação dos meandros em correntes de contorno confluentes. 0 estudo foi bem sucedido em explicar a formação do padrão de retroflexão, mas falhou em reproduzir as amplitudes dos meandros observadas, denotando que um processo de instabilidade geofísica era necessário para que estes crescessem. Em sequência, Silveira \& Flierl (2002) estudaram o papel da instabilidade baroclínica na formação de estruturas vorticais em CCOs confluentes num oceano de $2 \frac{1}{2}$ camadas. Verificaram então, a ocorrência de vórtices anticiclônicos, que se separavam do lóbulo de retroflexão, além de eventualmente estruturas ciclônicas em meandros subsequentes.

A configuração de $2 \frac{1}{2}$-camadas adotada por Silveira \& Flierl (2002), entretanto, filtra o modo barotrópico e a dinâmica fica restrita à interação dos dois primeiros modos baroclínicos. A escolha desta estrutura certamente se deve à dificuldade da implementação do modo barotrópico em configurações mais idealizadas, como os modelos DC. Entretanto, o papel deste modo dinâmico deve ser levantado. Consideremos novamente 0 exemplo da Confluência Brasil-Malvinas: ambas correntes possuem componente barotrópica importante na latitude de separação. A região da CB apresenta, além de níveis de superfície e picnoclínicos fluindo para o sul, também os níveis intermediário e profundo (Zemba, 1991). Já a CM é formada a partir de um ramo da Corrente Circumpolar Antártica que guina anticiclonicamente após deixar o Estreito de Drake (Tomczak \& Godfrey, 1993). A 
CM é portanto uma corrente profunda, também, com importante componente barotrópica no escoamento médio (Garzoli \& Garrafo, 1989).

0 objetivo principal deste trabalho é então investigar o papel da instabilidade baroclínica na formação de estruturas vorticais em Confluência de CCOs utilizando um modelo idealizado de duas camadas com os modos barotrópico baroclínico presentes no escoamento básico. Em particular, o modelo é baseado em Silveira \& Flier/(2002), e apresenta as seguintes características:

- Oceano semi-infinito, inercial, não-linear, e quase-geostrófico de duas camadas com superfície rígida e fundo plano. A estrutura vertical do modelo é obtida através da calibração apresentada em Flier/ (1978), de forma a aproximar os parâmetros dinâmicos relevantes (em particular, o raio de deformação baroclínico) do modelo de duas camadas aos parâmetros do oceano real (e continuamente estratificado) da região da Confluência Brasil-Malvinas.

- Convergência de duas CCOs na camada superior em uma dada latitude de separação arbitrariamente imposta, a partir da qual essas correntes formam um jato zonal que se propaga na direção leste-oeste.

- Campo de vorticidade potencial quase-geostrófica discretizado por duas regiões homogêneas, separadas por uma frente em cada camada. Assim 0 jato zonal na camada superior apresenta vorticidade potencial ciclônica (positiva) num lado da descontinuidade, e anticiclônica (negativa) no lado oposto.

- Dada a natureza teórica do estudo, o escoamento na camada inferior será variado para análise da variação das propriedades de instabilidade linear e não-linear do sistema de correntes, bem como suas consequências no desenvolvimento de vórtices de retroflexão.

\section{FORMULAÇÃO DO MODELO}

\section{A Estrutura Dinâmica do Modelo}

0 sistema físico escolhido para esse estudo é o sistema quasegeostrófico de duas camadas no plano $f$ (Figura 2), formulado por Pedlosky (1979), e derivado originalmente como um modelo de dois níveis por Phillips (1954). É composto por duas camadas imiscíveis de densidade constante $\rho$ e $\rho+\Delta \rho$, com $\Delta \rho \ll \rho$. 0 modelo quase-geostrófico de duas camadas incorpora então, a estratificação oceânica em sua forma mínima, onde a picnoclina é reduzida a uma interface separando duas camadas de densidade ligeiramente diferentes.

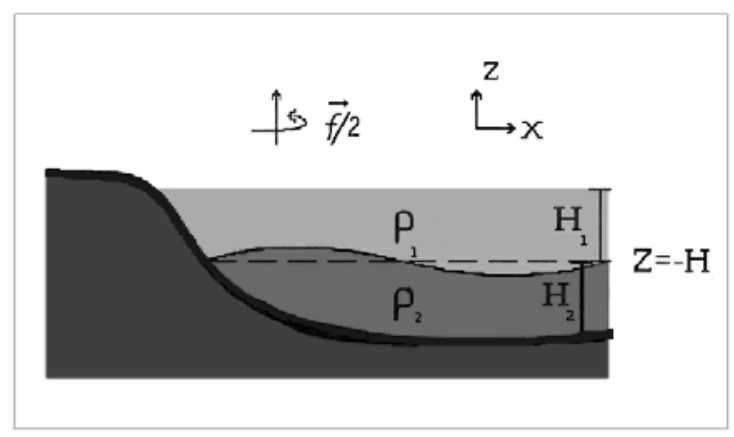

Figura 2 - Estrutura vertical do modelo quase-geostrófico de duas camadas, onde $H_{1}$ e $H_{2}$ são as alturas de repouso da primeira e segunda camadas respectivamente com $H=H_{1}+H_{2}$ e $\rho_{1} \rho_{2}$ são as densidades da primeira e segunda camadas.

Figure 2 - Vertical structure of the two layer quasigeostrophic model, where $\mathrm{H}_{1}$ and $\mathrm{H}_{2}$ are the stationary heights of the first and second layers respectively. $H=H_{1}+H_{2}$ and $\rho_{1}$ and $\rho_{2}$ are the densities of the first and second layers.

0 modelo apresenta número de Rossby $R o=\frac{U}{f L}$ pequeno comparado à unidade e, dessa forma, é geostrófico em primeira ordem.

Como a densidade é homogênea, o teorema de TaylorProudman é válido dentro de cada camada e, portanto, a velocidade não cisalha dentro desta. A dinâmica do sistema torna-se essencialmente bidimensional, sendo governada pela conservação de vorticidade potencial $q_{i}$, onde $i=1,2$ representa as camadas superior e inferior, respectivamente.

A estrutura de densidade simplificada a duas camadas equivale a uma filtragem modal, onde apenas dois primeiros modos dinâmicos (barotrópico e baroclínico) estão presentes. Faz-se necessária, então, a escolha da profundidade de repouso da interface $H_{1}$ e do salto de densidade entre as camadas $\epsilon$. Estes parâmetros de estrutura vertical serão determinados a partir da calibração de Flier/(1978), resumida no Apêndice.

\section{Dinâmica de Contornos}

A utilização da técnica semi-analítica de DC , do ponto de vista matemático, leva a uma redução concisa e elegante do sistema de equações diferenciais tratado, tornando possível soluções estacionárias analíticas e a evolução numérica do sistema, a partir de condições iniciais. Do ponto de vista físico, DC reduz os graus de liberdade do sistema não pela linearização da expressão de vorticidade potencial (VP) pela quase-geostrofia, mas pela redução de sua complexidade através da discretização do campo de VP: a dinâmica se restringe a acompanhar os movimentos dos contornos (ou frentes) de vorticidade (vide Pullin (1992) para mais detalhes). 
Tabela 1 - Escalas e Quantidades Adimensionais Correspondentes.

Table 1 - Corresponding scales and nondimensional quantities.

\begin{tabular}{|l|c|}
\hline Definição & Convenção \\
\hline $\begin{array}{l}\text { escala de profundidade } \\
\text { (=profundidade total) }\end{array}$ & $H=H_{1}+H_{2}$ \\
\hline espessura de repouso da camada & $h_{i}=H_{i} / H$ \\
\hline razão de aspecto entre as camadas & $\delta=h_{1} / h_{2}$ \\
\hline escala de densidade média & $\rho_{0}$ \\
\hline densidade da camada & $\rho_{i}$ \\
\hline salto de densidade normalizado & $\epsilon=\left(\rho_{2}-\rho_{1}\right) / \rho_{0}$ \\
\hline parâmetro de Coriolis & $f_{0}$ \\
\hline $\begin{array}{l}\text { escala de comprimento horizontal } \\
\text { (=raio de deformação interno) }\end{array}$ & $R_{d 1}$ \\
\hline parâmetro de acoplamento entre as camadas & $\gamma=R_{d 1}{ }^{2} f_{0}^{2} /\left(g \epsilon_{1} H_{1}\right)$ \\
\hline $\begin{array}{l}\text { escala de velocidade horizontal } \\
\text { (=velocidade máxima da primeira camada) }\end{array}$ & $\tilde{u}_{1}$ \\
\hline componentes das velocidades de camadas & $u_{i}, v_{i}$ \\
\hline escala da função de corrente & $R_{d 1} \tilde{u_{1}}$ \\
\hline função de corrente de camadas & $\psi_{i}$ \\
\hline função de corrente modal & $\Psi_{i}$ \\
\hline escala de tempo & $R_{d 1} \tilde{u_{1}}{ }^{-1}$ \\
\hline escala de vorticidade potencial & $R_{d 1}{ }^{-1} \tilde{u_{1}}$ \\
\hline vorticidade potencial de camadas & $q_{i}$ \\
\hline vorticidade potencial modal & $Q_{i}$ \\
\hline escala do salto de vorticidade potencial & $R_{i}$ \\
\hline salto vorticidade potencial de camadas & \\
\hline
\end{tabular}

Em nosso modelo, em cada uma das duas camadas, existe um contorno (descontinuidade) que separa duas regiões de VP de valores distintos. Como em DC, fontes e sorvedouros de VP não são possíveis, os contornos são linhas materiais, e a VP é conservada. A forma adimensionalizada da equação de conservação de VP, segundo a Tabela 1, é dada por

$$
\frac{d}{d t} q_{i}=\left[\frac{\partial}{\partial t}+u_{i} \frac{\partial}{\partial x}+v_{i} \frac{\partial}{\partial y}\right] q_{i}=0,
$$

tal que

$$
M_{i j} \psi_{j}=\left[\delta_{i j} \nabla^{2}+Z_{i j}\right] \psi_{j}=q_{i},
$$

onde $\psi_{j}$ é a função de corrente na j-ésima camada, $\delta$ é a razão de aspecto e $\gamma$ é 0 número de Froude da camada superior que pode ser entendido como o parâmetro de acoplamento entre as duas camadas e

$$
Z=\left[\begin{array}{cc}
-\gamma & \gamma \\
\delta \gamma & -\delta \gamma
\end{array}\right]
$$

representa a matriz de estrutura vertical cujos autovalores são $\lambda_{0}=0$ e $\lambda_{1}=\gamma(1+\delta)$ e correspondem ao inverso do quadrado dos raios de deformação barotrópico e baroclínico com autovetores

$$
\begin{aligned}
F_{i 0} & =[1,1] \mathrm{e} \\
F_{i 1} & =\left[\delta^{-1 / 2},-\delta^{1 / 2}\right],
\end{aligned}
$$

onde $F_{i m}$ representa a amplitude do m-ésimo modo dinâmico na i-ésima camada. $m=0$ correponde ao modo barotrópico, e $m=1$ ao modo baroclínico.

Para a utilização da DC, os jatos devem ter a estrutura de VP discretizada. Escolhendo a configuração com uma frente em cada 
camada tem-se

$$
q_{i}=q_{0 i}+\Delta_{i} \mathcal{H}\left(y-\bar{y}_{i}-\eta_{i}\right)
$$

onde $q_{0 i}$ é 0 valor de $q_{i}$ ao sul do contorno, $\Delta_{i}$ é 0 salto de vorticidade na i-ésima camada e $\mathcal{H}$ é a função de Heaviside. A posição média do contorno é $\bar{y}_{i}$, e $\eta_{i}$ representa a perturbação da frente na i-ésima camada, portanto, dependente do tempo.

Separando-se o campo $q_{i}$ em um estado básico e um estado perturbado, têm-se

$$
M_{i j}\left(\bar{\psi}_{j}, \psi_{j}^{\prime}\right)=\left(\bar{q}_{i}, q_{i}^{\prime}\right)
$$

com

$$
\begin{gathered}
\bar{q}_{i}=q_{0 i}+\Delta_{i} \mathcal{H}\left(y-\bar{y}_{i}\right) e 22( \\
q_{i}^{\prime}=\Delta_{i}\left[\mathcal{H}\left(y-\bar{y}_{i}-\eta_{i}\right)-\mathcal{H}\left(y-\bar{y}_{i}\right)\right]
\end{gathered}
$$

A evolução temporal do contorno na i-ésima camada é computada utilizando-se as equações lagrangeanas

$$
\begin{aligned}
\frac{d}{d t} x_{i} & =\overline{u_{i}}+u_{i}^{\prime} \\
\frac{d}{d t} \eta_{i} & =\bar{v}_{i}+v_{i}^{\prime}
\end{aligned}
$$

0 sistema lagrangeano não-linear é então resolvido calculando-se as soluções para os campos $\bar{\psi}_{i}$ e $\psi_{i}^{\prime}$, e as velocidades correspondentes são obtidas a partir da definição de função de corrente

$$
\left[u_{i}, v_{i}\right]=\left[-\frac{\partial \psi_{i}}{\partial y}, \frac{\partial \psi_{i}}{\partial x}\right] .
$$

Através da diagonalização da Eq. 2 em termos das amplitudes modais

$$
\begin{aligned}
\psi_{i}(x, y, z, t) & =\Psi_{m} F_{i m} \\
q_{i}(x, y, z, t) & =Q_{m} F_{i m},
\end{aligned}
$$

onde $i$ representa a i-ésima camada e $j, 0$ j-ésimo modo dinâmico, podemos podemos obter duas equações desacopladas que dependem da estrutura modal do sistema

$$
\nabla^{2} \Psi_{0}=Q_{0}
$$

e

$$
\left[\nabla^{2}-\gamma(1+\delta)\right] \Psi_{1}=Q_{1}
$$

onde os índices 0 e 1 representam, novamente, os modos barotrópico e baroclínico e esses respeitam a condição de ortonormalidade

$$
\delta_{m n}=h_{i} F_{i n} F_{i m} .
$$

\section{Os Campos de Função de Corrente e Velocidade Estacionários}

0 campo $\bar{\psi}_{i}$ é obtido resolvendo-se as Eqs. 14 e 15 por iteração numérica, uma vez estabelecidas as condições de contorno. 0 padrão de confluência é bastante complexo e a obtenção de uma solução analítica não é possível .

As condições de contorno para o estado estacionário são então escritas em termos das amplitudes modais

$$
\Psi_{m}=\left(F^{-1}\right)_{m i} \psi_{i}
$$

No contorno oeste,

$$
\bar{\Psi}_{m}(0, y)=0
$$

para $m=0,1$ e representa a condição de fluxo normal à costa nulo. Nas demais bordas, as condições para a amplitude do modo baroclínico seguem Silveira et al. (1999) e são dadas por

$$
\begin{aligned}
\bar{\Psi}_{1}(x, \pm \infty) & =\mp\left(F^{-1}\right)_{1 i} \frac{\Delta_{i}}{2}\left(1-e^{-x}\right) \\
\bar{\Psi}_{1}(\infty, y) & =\left(F^{-1}\right)_{1 i} \frac{\Delta_{i}}{2}\left(1-e^{-|y|}\right) .
\end{aligned}
$$

Já para o modo barotrópico, propomos a configuração de escoamento definida nas bordas norte, sul e leste por

$$
\begin{gathered}
\bar{\Psi}_{0}\left(x \leq r_{0}, \pm r_{0}\right)=\mp\left(F^{-1}\right)_{0 i} \frac{\Delta_{i}}{8}\left(x^{2}-2 r_{0} x\right), \\
\bar{\Psi}_{0}\left(x>r_{0}, y\right)=\left(F^{-1}\right)_{0 i} \frac{\Delta_{i}}{8}\left(y^{2}-2 r_{0}|y|\right) .
\end{gathered}
$$

onde $r_{0}$ é um parâmetro arbitrário que determina a escala do efeito da borda oeste no escoamento barotrópico. Neste trabaIho, consideramos, após estudos de sensibilidade $r_{0}=20$ raios de deformação e esta distância representa também a extensão do domínio meridional do modelo. Esta escolha de amplo valor de $r_{0}$ equivale a avaliar a função de corrente baroclínica no $\infty$, onde 0 efeito da presença do contorno oeste no escoamento não é mais sentido

A Figura 3 exibe os campos de $\bar{\Psi}_{0}$ (painel esquerdo) e $\bar{\Psi}_{1}$ (painel direito). Com a obtenção de $\bar{\Psi}_{m}, \bar{\psi}_{i}$ é determinado por mapeamento inverso e as velocidades $\overline{u_{i}}$ e $\overline{v_{i}}$ são computadas utilizando-se a aproximação de diferenças finitas de acordo com a Eq. 11. 
Amplitude da função de corrente barotrópica $\times 0.01$

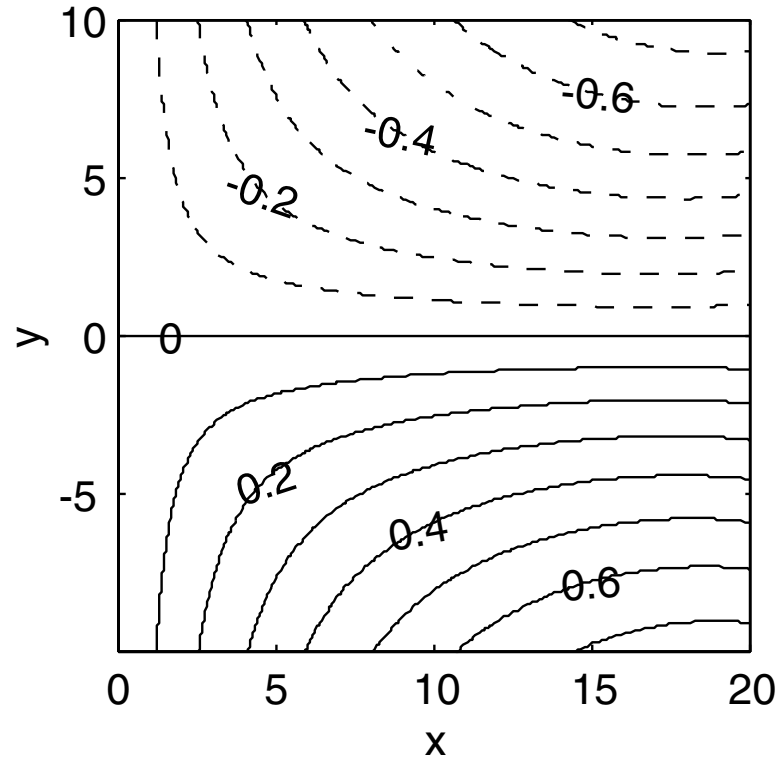

(a)

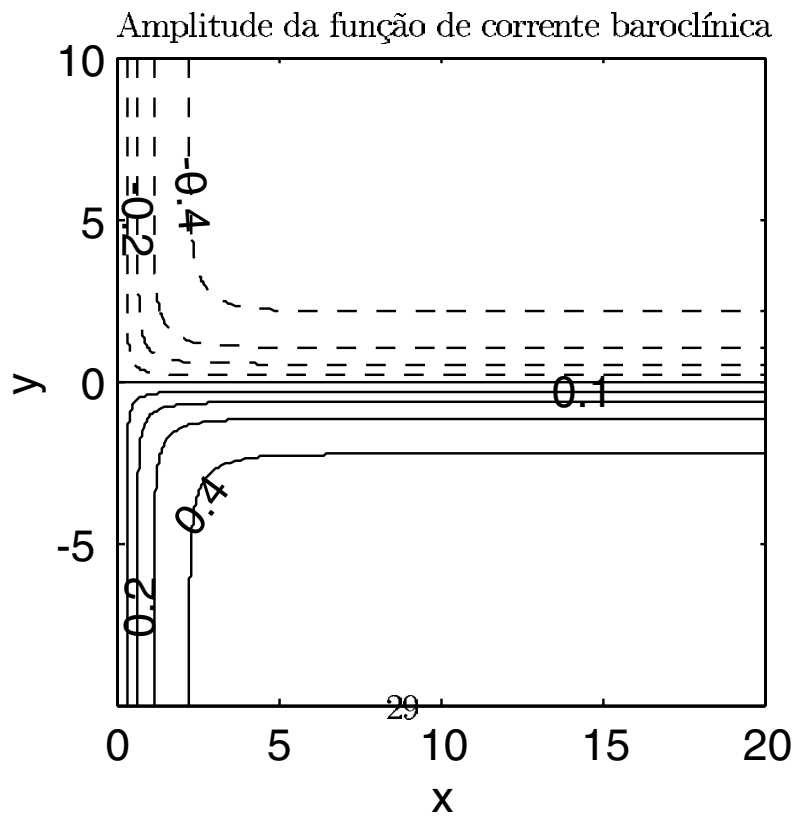

(b)

Figura 3 - Amplitude da função de corrente para os modos barotrópico (painel esquerdo) e baroclínico (painel direito). As linhas tracejadas indicam valores negativos e as linhas sólidas valores positivos.

Figure 3 - Amplitude of the stream function for the barotropic (left panel) and baroclinic (right panel) mode. The dotted lines indicate negative values and the solid lines positive values.

\section{Os Campos de Função de Corrente e Velocidade Perturbados}

A função de corrente perturbada $\psi_{i}^{\prime}$ é calculada por

$$
\psi_{i}^{\prime}(x, y, t)=\Delta_{j} \iint_{A_{j}} G\left(x, y \mid x^{\prime}, y^{\prime}\right) d x^{\prime} d y^{\prime},
$$

onde as funções de Green, que respeitam a condição de contorno $\psi^{\prime}(0, y, t)=0$, são dadas por

$$
G_{i j}=-h_{i} F_{i m} F_{j m} \frac{1}{2 \pi}\left[K_{0}\left(\gamma_{m} r_{+}\right)-K_{0}\left(\gamma_{m} r_{-}\right)\right] .
$$

A função $G_{i j}$ representa a influência de uma perturbação da j-ésima camada na i-ésima camada e suas variáveis são dadas por

$$
r_{+}=\sqrt{\left(x-x^{\prime}\right)^{2}+\left(y-y^{\prime}\right)^{2}}
$$

e

$$
r_{-}=\sqrt{\left(x-x^{\prime}\right)^{2}+\left(y-y^{\prime}\right)^{2}} .
$$

A função de Green para $m=0$ (modo barotrópico), corresponde a $\gamma_{0} \rightarrow 0$, e dessa forma $K_{0}\left(\gamma_{0} r\right) \rightarrow-\ln (r)$.

A expressão para as velocidades perturbadas é obtida a partir da combinação das Eqs. 11 e 22 e utilização do Teorema de Green. Uma adicional integração por partes, recomendada por Po/vaniet al. (1989), minimiza problemas de singularização para pequenos argumentos das funções de Green, e resulta em

$$
\begin{gathered}
{\left[u_{i}^{\prime}, v_{i}^{\prime}\right]=} \\
-\Delta_{j} \int_{\partial A_{j}} \frac{\partial G_{i j}(r)}{\partial r}\left[\left(x-x^{\prime}\right),\left(y-y^{\prime}\right)\right] d r .
\end{gathered}
$$

\section{Evolução Numérica do Sistema}

Essencialmente, 0 algoritmo DC rastreia o contorno (ou a frente de VP) como um conjunto de parcelas de fluido lagrangeanas, avançando cada uma invididualmente pela sistema de Eqs. 10. Estas são discretizadas utilizando um esquema de Runge-Kutta de segunda ordem, após a avaliação das velocidades. As componentes de velocidade estacionária são interpoladas dentro da matriz confinada pelos limites de $\pm r_{0}$ tanto na extensão meridional como zonal. Para limites de valores zonais que excedam $x=r_{0}$, são utilizadas as expressões analíticas de jatos infinitos dadas pelas Eqs. 19 e $21 \mathrm{em}$ termos modais. As velocidades perturbadas são avaliadas pela aproximação numérica da Eq. 24 de acordo com Zou et al. (1988). Um esquema simples de rearranjo das parcelas de fluido (inserção e eliminação) mantém a frente com resolução pré-definida que garante a regularização das 
integrais envolvendo as funções de Bessel e logarítmicas. Por ser um domínio semi-infinito, técnicas como a Cirurgia de Contornos (Dritschel, 1989), que permitem o destacamento de vórtices do eixo das correntes não podem ser empregadas facilmente: estas requerem condições de contorno periódicas. Assim, a integração do modelo é conduzida até 0 isolamento da primeira estrutura vortical.

\section{Modelo Linear}

Num sistema de escoamentos não-paralelos como o padrão de confluência, a avaliação de propriedades de instabilidade linear, tais como taxas de crescimento das amplitudes das ondas, velocidade de fase e de grupo não é trivial. Em contra-partida, existe vasto conhecimento teórico acerca de escoamentos paralelos, em particular, jatos infinitos zonais. Utilizaremos nesse trabalho a avaliação dessas propriedades ao jato zonal resultante da confluência para orientar a escolha das condições iniciais no intento de seu conteúdo espectral conter sistemas bastante instáveis. Adicionalmente utilizamos as relações lineares para determinar dos saltos de vorticidade a partir dos valores de velocidade nas frentes.

Primeiramente, linearizamos as Eqs. 7 e $10 \mathrm{em}$ torno de $\bar{y}_{i}$, considerando soluções proporcionais a $e^{i k(x-c t)}$. obtém-se então o problema de autovalores dado por

$$
\begin{aligned}
M_{i j}{ }^{k} \psi_{j}^{\prime}\left(\overline{y_{j}}\right) & =-\Delta_{i} \eta_{i} \delta\left(y-\bar{y}_{j}\right) \quad \mathrm{e} \\
M_{i j}{ }^{0} \overline{u_{j}}\left(\overline{y_{j}}\right) & =-\Delta_{i} \delta\left(y-\overline{y_{j}}\right)
\end{aligned}
$$

com

$$
M_{i j}{ }^{k}=\delta_{i j}\left(\frac{\partial}{\partial y^{2}}-k^{2}\right)+Z_{i j},
$$

e

$$
\bar{u}_{i}\left(\bar{y}_{i}\right) \eta_{i}-c \eta_{i}=\psi_{i}^{\prime}\left(\bar{y}_{i}\right)
$$

As funções de Green para o caso linear tomam a forma

$$
\left(G^{k}\right)_{i j}=-h_{i} F_{i 1} F_{j 1} \frac{1}{2 \sqrt{k^{2}+\gamma^{2}}} e^{\sqrt{k^{2}+\gamma^{2}}\left|y-y^{\prime}\right|}
$$

para o modo baroclínico e

$$
\left(G^{k}\right)_{i j}=h_{i} F_{i 0} F_{j 0} \frac{1}{2}\left[|y|-r_{0}\right]
$$

para o modo barotrópico.

Uma vez calculadas as funções de Green para o modelo linear, pode-se utilizar as Eqs. 25 para obtenção dos campos $\psi_{i}^{\prime}(y, t)$ e $\overline{u_{i}}(y)$ na forma

$$
\begin{aligned}
\psi_{i}^{\prime}(y, t) & =G^{k}{ }_{i j}\left(y \mid \bar{y}_{j}\right) \Delta_{j} \eta_{j}, \\
\overline{u_{i}}(y) & =G^{k}{ }_{i j}\left(y \mid \bar{y}_{j}\right) \Delta_{j}
\end{aligned}
$$

Obtidos $\psi_{i}^{\prime}(y, t)$ e $\overline{u_{i}}(y)$, esses campos podem ser avaliados em $\bar{y}_{j}$ e utilizando a Eq. 27 podemos encontrar a velocidade de fase $c$ das ondas linearmente instáveis.

\section{RESULTADOS E DISCUSSÃO}

\section{Parâmetros de Estrutura Vertical}

Os parâmetros de estrutura vertical foram escolhidos com base em critérios quantitativos dados pela técnica de calibração dinâmica proposta por Flierl (1978). A técnica de calibração dinâmica, resumida no apêndice deste trabalho, impõe que a estrutura vertical do oceano de camadas quase-geostrófico seja determinada por um conjunto de grandezas que são matematicamente tratadas como funcionais da estratificação do oceano real.

Para determinar a estrutura vertical do modelo utilizamos vaIores climatológicos de salinidade e temperatura, com resolução de $1 / 4^{\circ}$, extraídos da climatologia atualizada Levitus \& Boyer (1998) da área compreendida entre $35^{\circ}-40^{\circ} \mathrm{S}$ e $50^{\circ}-60^{\circ} \mathrm{W}$. 0 raio de deformação e estrutura vertical do primeiro modo baroclínico são determinados de acordo com Houry et al. (1987) utilizando o perfil médio de densidade (e a frequência de Brunt-Vaisalla). Estes consistem nas curvas tracejadas apresentadas na Figura 4. A calibração realizada fornece como resultado $H_{1}=1366 \mathrm{~m} \mathrm{e}$ $\delta=0,436$ (com $H=H_{1}+H_{2}=4500 \mathrm{~m}$ ), bem como $\epsilon=8,4510^{-4}$, com $\rho_{0}=1024 \mathrm{~kg} / \mathrm{m}^{3}$.

A estrutura do modo discreto, calculada a partir da Eq. 5 , é apresentada na Figura 4

\section{Experimentos com Modelo de Dinâmica de Contornos}

As velocidades-núcleo nas duas camadas são $\overline{u_{1}}=1$, para todos os casos estudados enquanto as velocidades na camada 2 são dadas por $\overline{u_{2}}=(-0,3,-0,5,-0,65)$.

Na configuração básica do escoamento dos três experimentos, as CCOs convergem na camada superior para formar um jato zonal inercial que flui para leste. Há contrafluxos nas bordas. Já o escoamento na camada inferior é dominantemente para oeste, divergindo na fronteira oeste, com domínio da componente barotrópica.

É possível que 0 leitor indague por que utilizar uma camada inferior retrógrada, se evidências observacionais apontam padrões de correntes de contorno diminuindo com a profundidade, mas sem inversão de sentido ao longo da coluna de água. A resposta é que a estrutura dinâmica do sistema, e não sua estrutura cinemática, é o fator determinante para sua evolução. A estrutura dos modos dinâmicos dita a escolha do transporte nas 


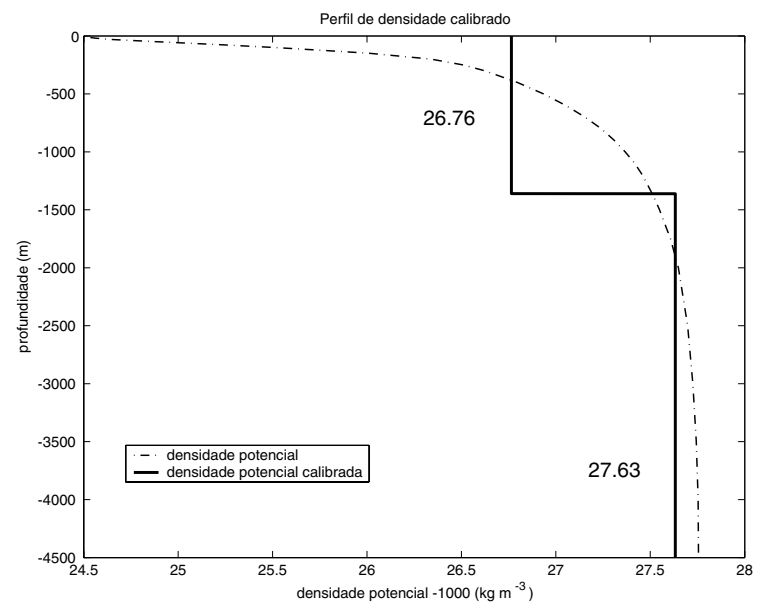

(a)

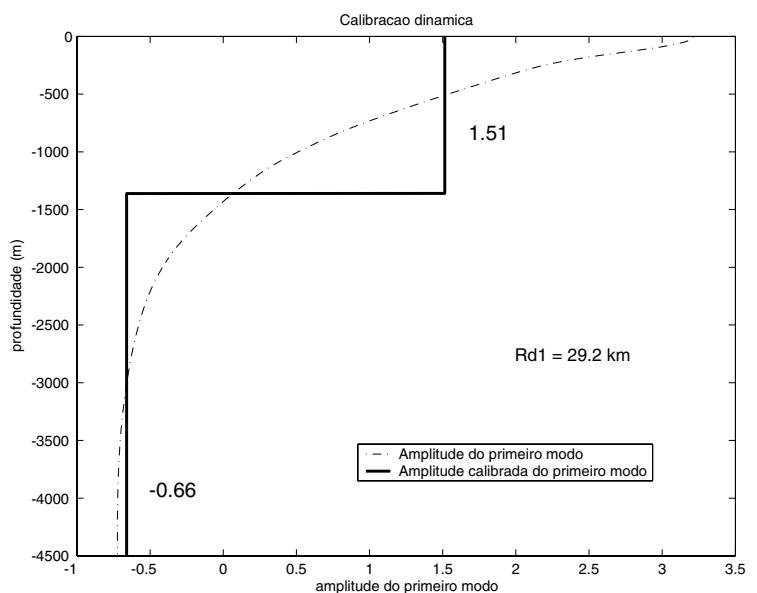

(b)

Figura 4 - Resultados da Calibração Dinâmica. Painel esquerdo: perfil de densidade média climatológica da região da Confluência Brasil-Malvinas (curva tracejada) e perfil de densidade calibrado para modelo de duas camadas (curva sólida). Painel direito: Estrutura do primeiro modo baroclínico climatológico (curva tracejada) e 0 modo baroclínico discreto do modelo de duas camadas (curva sólida). 0 valor do raio de deformação baroclínico é comum à climatologia do oceano real e ao modelo de duas camadas dinamicamente calibrado.

Figure 4 - Results of the Dynamic Calibration. Left panel: mean climatological density profile of the Brazil-Malvinas confluence (dotted curve) and density profile calibrated for the two layer model (solid curve). Right panel: structure of the first climatological baroclinic mode (dotted curve) and the discreet baroclinic mode for the two layer model (solid curve). The value for the deformation radius is common to both the climatology of the real ocean and the two layer model dinamically calibrated.

camadas. Essa escolha de abordagem propicia também a escoIha de velocidades mais realísticas para a camada superior, pois 0 surgimento de instabilidades depende diretamente de um valor crítico da velocidade de cisalhamento entre as camadas ( $P e^{-}$ dlosky, 1979). Em outras palavras, a estrutura vertical simplificada e idealizada do modelo de duas camadas requer um cisaIhamento muito intenso para reproduzir o efeito dinâmico de estrutura de densidade (e em quase-geostrofia, também velocidade) de distribuição contínua.

\section{Modelo Linear}

Nas Figuras 5, 6 e 7 são apresentadas as propriedades de instabilidade linear obtidas para os estudos de casos: $\overline{u_{2}}=$ $(-0,3,-0,5,-0,65)$, respectivamente, mostrando as características das ondas instáveis e sua tendência de propagação para 0 oceano calibrado dinamicamente.

A Figura 5, representa o caso em que a velocidade na camada inferior é $\overline{u_{2}}=-0,3$, mostrando que ondas instáveis propagam fase para leste com velocidades máximas em torno de 0, 15 e taxa de crescimento máxima das ondas instáveis de $\sigma=0,11$ para $k=0,7$.

Para 0 caso em que variamos a velocidade da camada inferior para $\overline{u_{2}}=-0,5$, a Figura 6 mostra que são geradas ondas linearmente instáveis quase-estacionárias com baixíssimas velocidades para leste com $k \geq 0,5$. A taxa de crescimento máxima das ondas instáveis é $\sigma=0,13$ para $k=0,68$, mostrando um leve aumento da taxa máxima de crescimento das ondas instáveis centrada em um comprimento de onda pouco maior que no caso anterior.

Ao variarmos novamente a velocidade na camada inferior fazendo desta vez $\overline{u_{2}}=-0,65$, e portanto, aumentando ainda mais o cisalhamento entre as camadas, a Figura 7, mostra ondas linearmente instáveis que propagam fase para oeste com velocidades máximas de $-0,1$. A taxa de crescimento máxima destas ondas é dada por $\sigma=0,14$ para $k=0,69$, evidenciando que as ondas tornam-se mais instáveis para este caso, embora a taxa máxima de crescimento continue praticamente no mesmo número de onda do caso anterior.

É importante notar que nos três casos estudados as ondas infinitamente longas são estáveis. Silveira and Flier/(2002) mostraram para o modelo de $2 \frac{1}{2}$ camadas que o único caso em que ondas infinitamente longas são estáveis ocorre quando $\left|h_{1} \Delta_{1}\right|=$ $\left|h_{2} \Delta_{2}\right|$. Para o modelo de duas camadas, essa condiç̧ão implica que $\left|\Delta_{1}\right|=\delta\left|\Delta_{2}\right|$ ou seja, a amplitude do modo barotrópico seria nula e 0 jato básico puramente baroclínico.

Os resultados descritos pelo modelo linear serão utilizados como guia do comportamento das ondas instáveis na 

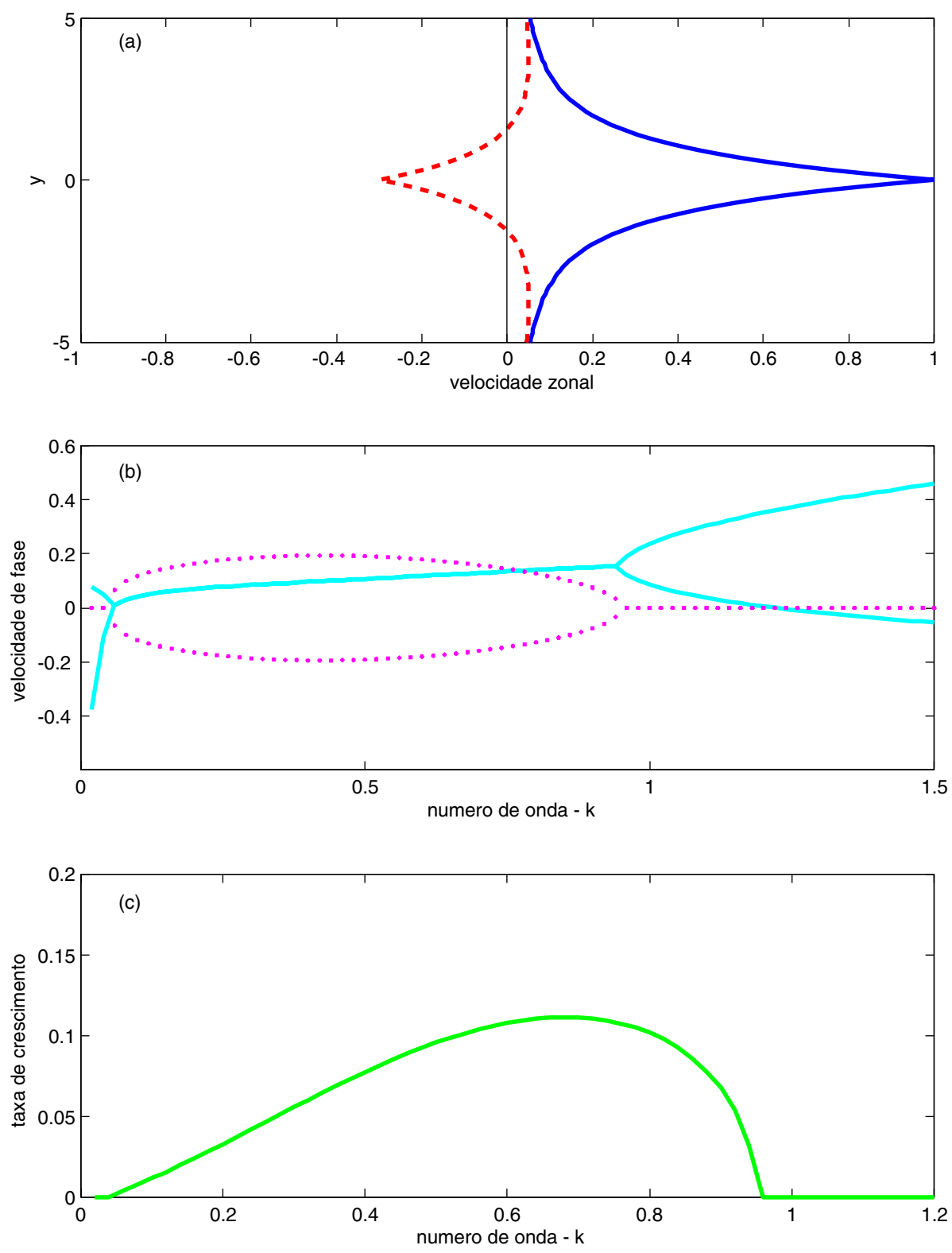

Figura 5 - Resultados do modelo linear para o oceano calibrado e $u_{2}=-0.3$. a) perfis dos jatos básicos para a camada superior (linha contínua) e camada inferior (linha tracejada). b) porções real ( $c_{r}$, linha contínua) e imaginária ( $c_{i}$, linha tracejada) da velocidade de fase. c) taxas de crescimento $\left(\sigma=k c_{i}\right)$.

Figure 5 - Results of the linear model for the calibrated ocean and $u_{2}=-0.3$. a) profiles for the basic jets of the upper layer (continuous line) and the lower layer (dotted line). b) real ( $c_{r}$, continuous line) and imaginary $\left(c_{i}\right.$, dotted line) portions of the phase velocity. c) growth rates $\left(\sigma=k c_{i}\right)$.

condução dos experimentos não-lineares auxiliando na definição das condições iniciais.

\section{Experimentos com Modelo Não-Linear}

A seguir são apresentados os resultados obtidos pelos experimentos CBM1 $\left.\overline{\left(\overline{u_{2}}\right.}=-0.3\right), C B M 2\left(\overline{u_{2}}=-0.5\right) \mathrm{e}$ $C B M 3\left(\overline{u_{2}}=-0.65\right)$ através da utilização do modelo DC.
A título de exemplo, a Figura 8 exibe os campos $\bar{\psi}_{i}$, obtidos através das amplitudes $\overline{\Psi_{m}}$, que representam a componente associada à posição não perturbada da frente. Este corresponde ao experimento $C B M 3$.

A Figura 9 apresenta a evolução temporal das frentes de VP para 0 experimento $C B M 1$ para 0 qual o modelo linear indica que ondas instáveis propagam fase para leste. 0 sistema foi per- 

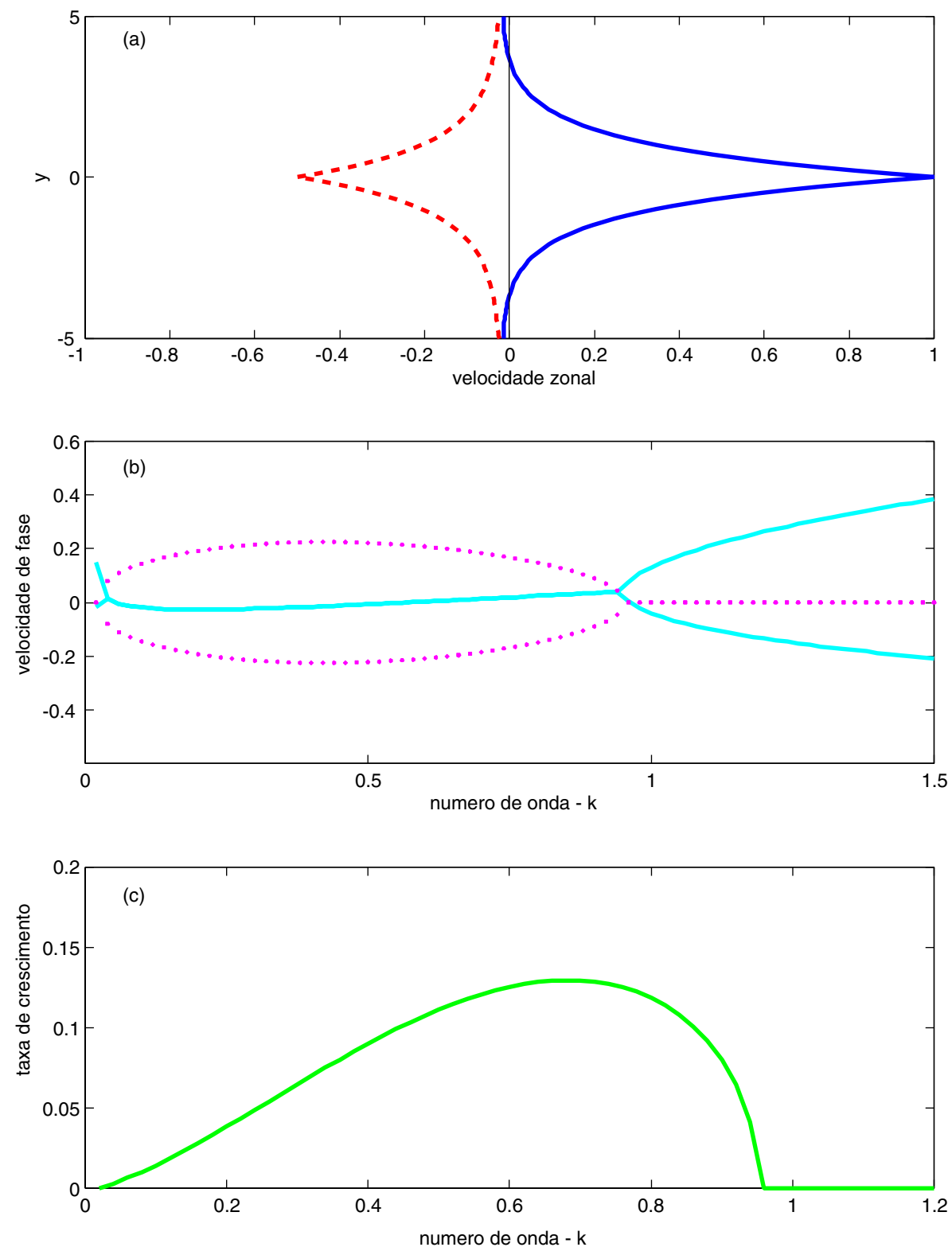

Figura 6 - Análogo à Figura 5 , mas para $u_{2}=-0.5$.

Figure $\mathbf{6}$ - Analogue to Figure 5 , only for $u_{2}=-0.5$.

turbado por um meandro gaussiano horário, com amplitude e largura de 3 raios de deformação, junto ao contorno e à confluência $(\mathrm{t}=0)$. À medida que 0 sistema evolui ( $\mathrm{t}=15)$, há a propagação para norte da perturbação na segunda camada fazendo com que as ondas fiquem fora de fase e os mecanismos de instabilidade baroclínica comecem a agir. É através do fechamento de fase das ondas instáveis, assinatura do processo de conversão baroclínica, que ocorre a formação de meandros, cujas amplitudes crescem temporalmente ( $\mathrm{t}=30)$. Esses meandros formam um dipolo baroclínico a sul da posição média do contorno, que se destacará do eixo principal da corrente em $x \geq 5$ ( $t=40)$. Ou seja, um dipolo baroclínico que é antihorário na camada superior é emitido a $x=5$. A instabilização do sistema conduz a um padrão semelhante ao da retroflexão, mas com a emissão dos vórtices ocorrendo a partir do cavado primário.

A Figura 10 apresenta a evolução temporal das frentes de VP 

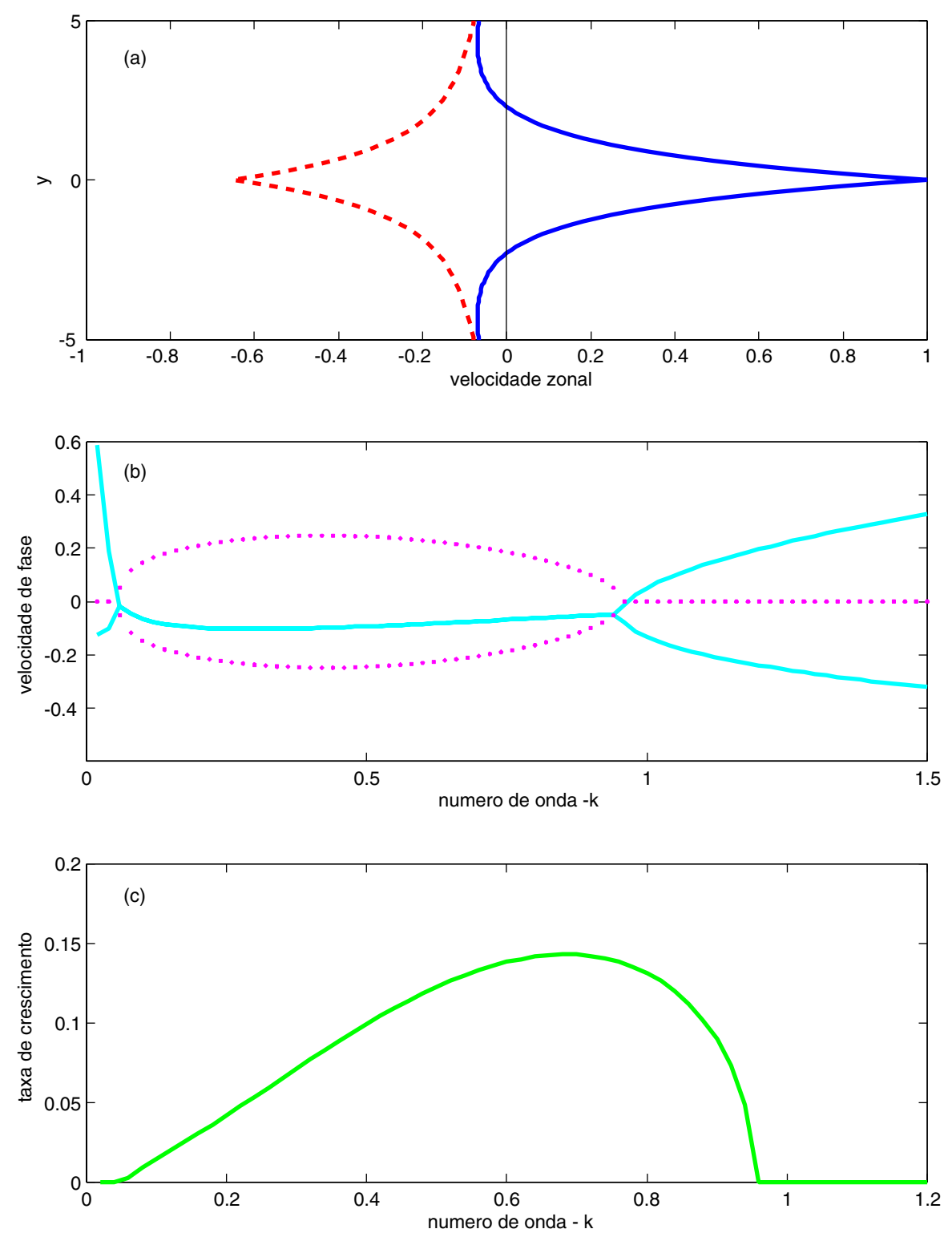

Figura 7 - Análogo à Figura 5 , mas para $u_{2}=-0.65$.

Figure 7 - Analogue to Figure 5 , only for $u_{2}=-0.65$.

para o experimento $C B M 2$ para 0 qual 0 modelo linear indica que ondas instáveis quase-estacionárias. A mesma perturbação do experimento $C B M 1$ foi usada aqui por conter potência no número de onda linearmente mais instável. À medida que o sistema evolui ( $\mathrm{t}=20)$, há novamente propagação para norte da perturbação na segunda camada fazendo, com que as ondas fiquem fora de fase ocorrendo fechamento de fase das ondas instáveis, proporcionando a formação de meandros instáveis $(\mathrm{t}=30)$. Esses meandros formam um dipolo baroclínico, ou seja, um dipolo com vorticidades de sinais diferentes em cada camada, a sul da posição média do contorno que se destaca do eixo principal da corrente em $x \geq 5$ ( $\mathrm{t}=40)$. À norte da posição média do contorno, o lóbulo formado na camada 2 é comprimido contra 0 contorno e passa a sentir o efeito da parede, enquanto na camada superior, devido à quase-estacionaridade das ondas instáveis, 0 lóbulo formado encontra-se próximo à costa.

0 padrão de instabilidade obtido nos experimentos $C B M 1$ e $C B M 2$ é similar, porém os meandros formados no expe- 


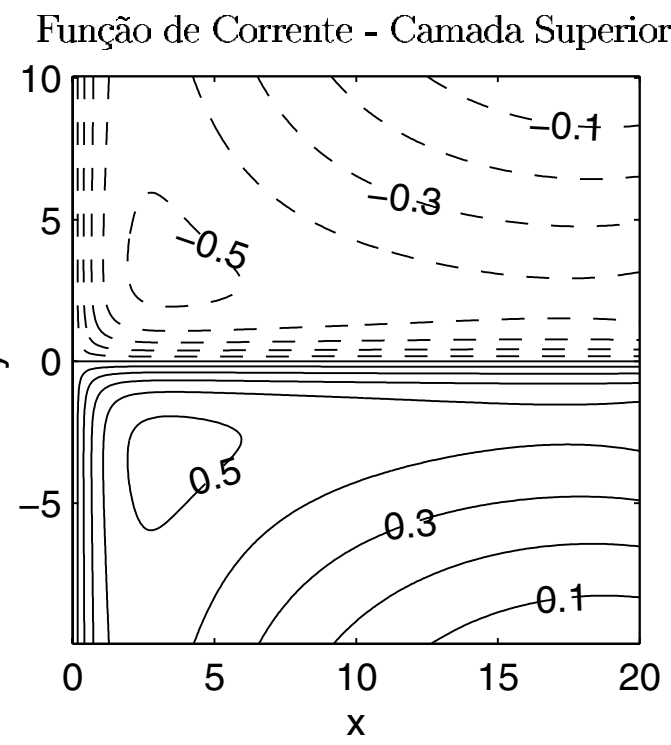

(a)

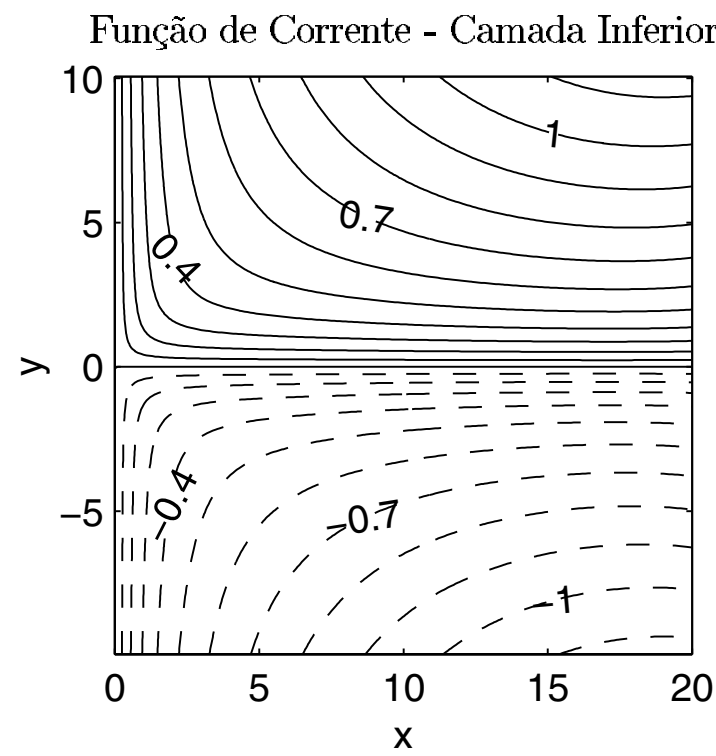

(b)

Figura 8 - Amplitude da função de corrente para a camada superior (painel esquerdo) e inferior (painel direito) para 0 experimento CBM3 $\left(u_{2}=-0.65\right)$. As linhas tracejadas indicam valores negativos e as linhas sólidas valores positivos.

Figure 8 - Amplitude of the stream function for the upper (left panel) and lower (right panel) layer for the CBM3 experiment ( $u_{2}=-0.65$ ). The dotted lines indicate negative values and the solid lines, positive values.

rimento $C B M 2$ apresentam desenvolvimento mais evidente e mais rápido que no experimento $C B M 1$, concordando com os resultados do modelo linear. Além deste fato, as ondas instáveis praticamente não se propagam no experimento $C B M 2$. Portanto, embora os padrões sejam similares nos dois experimentos, a diferença entre as velocidades de fase das ondas instáveis faz com que no experimento $C B M 1$ o meandramento apresente comprimentos de onda pouco mais curtos e a formação e emissão do dipolo ocorra mais lentamente que no experimento $C B M 2$.

0 último caso e também 0 mais dinamicamente é apresentado no experimento $C B M 3$ (Figura 11), onde resultados obtidos pelo modelo linear indicam que ondas instáveis propagam-se para oeste.

0 sistema é novamente perturbado por um meandro gaussiano junto ao contorno oeste. Assim que o modelo começa a evoluir $(t=0)$, o meandro na camada inferior é deslocado a norte $(t=20)$. Isto impõe ao sistema uma diferença de fase entre as ondas instáveis das duas camadas, próximo ao contorno, fazendo com que os mecanismos de instabilidade baroclínica passem a agir. Esses mecanismos, aliados à deriva para oeste das ondas instáveis propiciam a formação de um dipolo baroclínico que na camada superior é antihorário $(t=30)$. Uma vez próximo à costa, o dipolo interage com o contorno via efeito imagem (que consiste na eliminação do contorno e na introdução de um dipolo de mesma intensidade e sinal oposto, simetricamente posicionado em relação à linha de costa) e pode ser eventualmente advectado para sul $(t=40)$. 0 modelo fica singular quando da emissão do dipolo e a integração precisa ser interrompida. Tal estrutura bipolar, na primeira camada, é bastante semelhante àquelas da Confluência Brasil-Malvinas, com o lóbulo anticiclônico, formando anticiclones junto à margem continental (Olson et al., 1988). 0 experimento C BM3, portanto, indica que a presença de ondas instáveis que se propagam para oeste leva à formação e emissão de vórtices e dipolos juntos ao contornos, 0 que não ocorreu para os dois casos anteriores.

\section{SUMÁRIO E CONCLUSÕES}

Neste estudo, procedemos à investigação da dinâmica de mesoescala da confluência de duas correntes de contorno oeste num oceano de duas camadas quase-geostrófico, não viscoso e inercial no plano $f$. Buscou-se o isolamento do efeito da instabilidade baroclínica e a compreensão do papel dinâmico do modo barotrópico no escoamento básico do sistema hidrodinâmico. Para tanto, utilizamos a técnica de dinâmica de contornos, onde a estrutura de vorticidade potencial é simplificada (em outras palavras, discretizadas em função degrau tal qual a estrutura de massa). A estrutura vertical do modelo é obtida através de calibração do modelo de duas camadas em relação á estrutura 

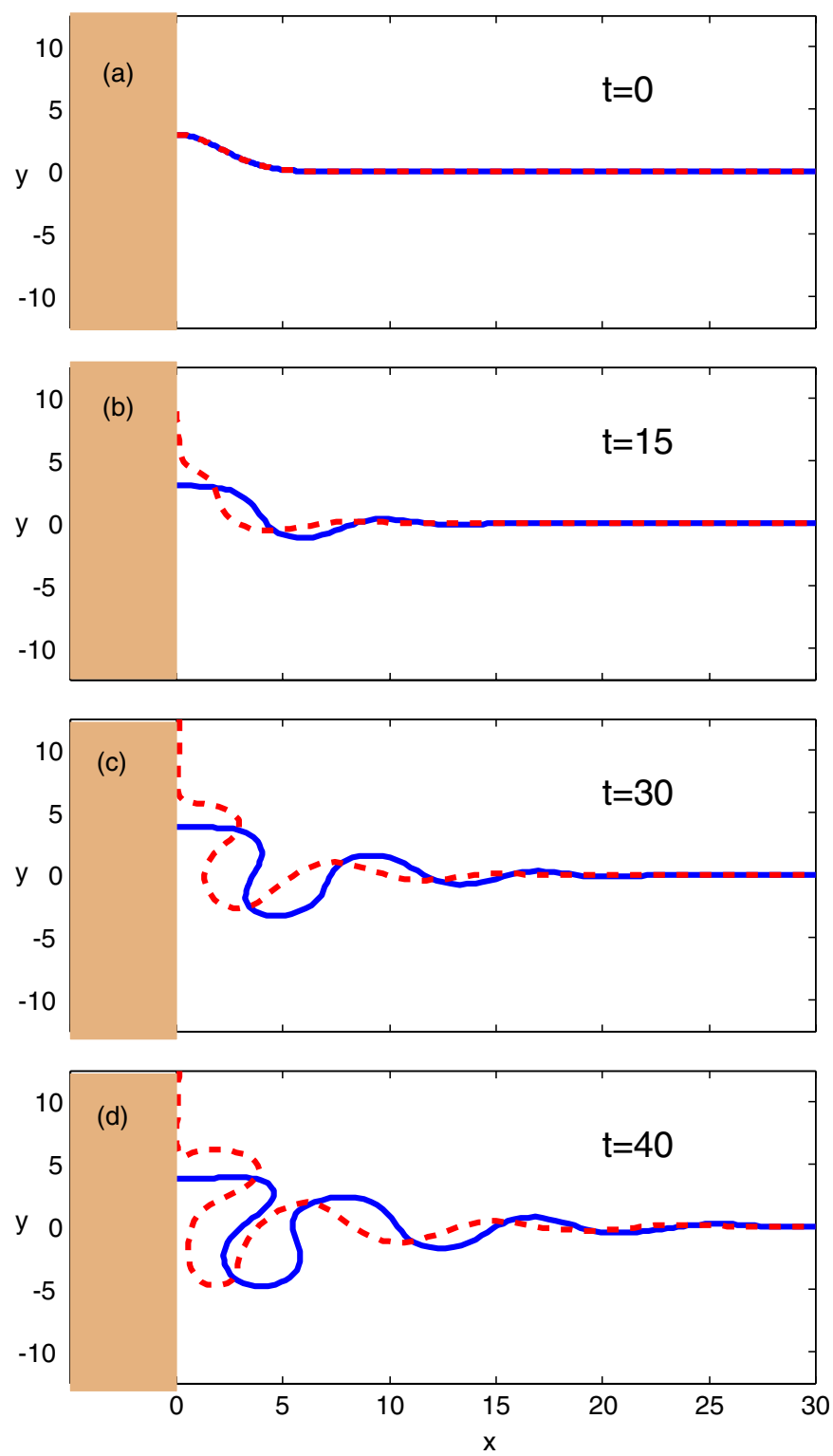

Figura 9 - Resultados do modelo não-linear para o oceano calibrado e $u_{2}=-0.3$, experimento C BM 1. A linha sólida representa a primeira camada e a linha tracejada a segunda camada.

Figure 9 - Results of the non-linear model for the calibrated ocean and $u_{2}=-0.3$, experiment C BM1. The solid line represents the first layer and the dotted line, the second layer.

modal da região da Confluência Brasil-Malvinas. Tal estrutura modal foi estimada a partir da climatologia da área compreendida entre $35^{\circ}-40^{\circ}$ S e $50^{\circ}-60^{\circ} \mathrm{W}$.

Dois modelos de dinâmica de contornos foram desenvolvidos. Estes apresentam um sistema de correntes de contorno oeste com transporte simétrico e duas frentes de vorticidade, uma por camada. 0 escoamento na camada superior é sempre convergente formando um jato zonal para leste. Para os casos aqui discuti- dos, o escoamento na camada inferior é divergente, com jato zonal de velocidades dominantemente para oeste. Analiticamente, uma configuração original de escoamento básico barotrópico é proposta, pois trabalhos anteriores envolvendo DC em escoamentos não paralelos como Silveira etal. (1999) e Silveira \& Flier/(2002), não se utilizam do modo barotrópico.

0 modelo linear determina as propriedades de instabilidade dos jatos zonais básicos resultantes da convergência de duas cor- 

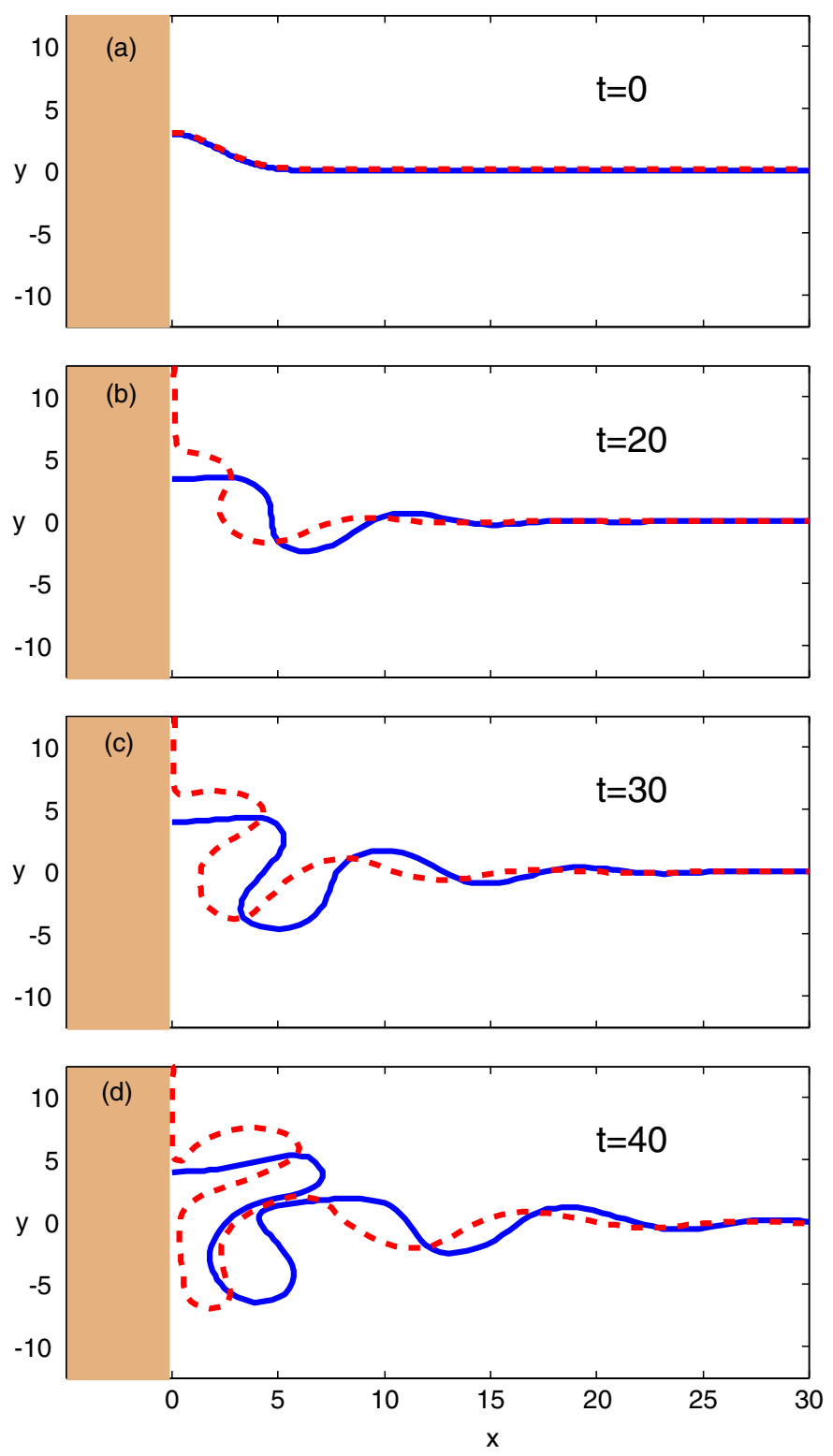

Figura 10 - Análogo à Figura 9 , mas para 0 experimento $C B M 2$, onde $u_{2}=-0.5$.

Figure 10 - Analogue to Figure 9 , only for experient $C B M 2$, where $u_{2}=-0.5$.

rentes de contorno. 0 modelo não linear permite a evolução temporal do sistema, através do acompanhamento dos movimentos das frentes de vorticidade.

Os resultados dos experimentos mostram que a presença confluência e da fronteira meridional favorecem ondas longas como indicado por Silveira et al. (1999), adicionando-se a isso o fato que a presença do modo barotrópico no jato básico, por si só, também causa a seleção de ondas linearmente mais longas. Isto é evidente ao compararmos os resultados das simulações deste trabalho com aquelas de Silveira \& Flierl(2002) para $2 \frac{1}{2}$ camadas.

A inclusão do modo barotrópico no modelo DC não afetou significativamente 0 comportamento qualitativo do sistema em relação ao trabalho de Silveira \& Flierl(2002). As taxas de crescimento aqui obtidas mostraram-se menores e os comprimentos de onda instáveis maiores que os de Silveira \& Flier/(2002). Pode-se argumentar que os parâmetros utilizados não são exatamente os mesmos, mas testes de sensibilidade do modelo com 

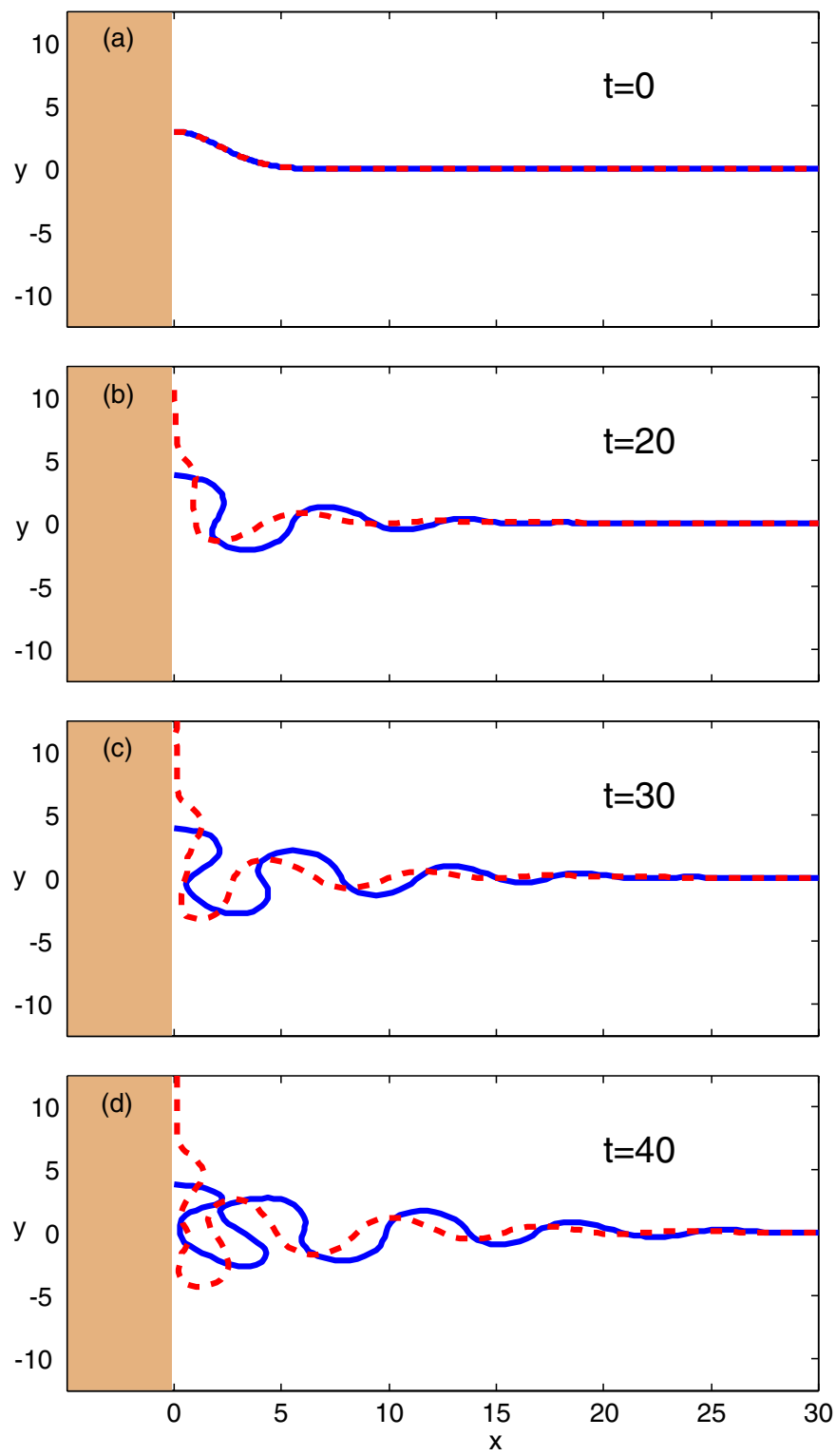

Figura 11 - Análogo à Figura 9, mas para 0 experimento $C B M 3$, onde $u_{2}=-0.65$.

Figure 11 - Analogue to Figure 9 , only for experient C BM3, where $u_{2}=-0.65$.

os parâmetros utilizados por esses autores mostraram o mesmo comportamento para as propriedades de instabilidade. 0 fato do modelo de 2 camadas apresentar taxas de crescimento menores que o modelo de $2 \frac{1}{2}$ camadas de Silveira \& Flier/ (2002) é interpretado como sendo resultado da presença de apenas um modo baroclínico no sistema. Da mesma forma, o fato de ondas mais longas serem instáveis é resultado da presença do modo barotrópico no sistema.

De fato nos casos estudados as ondas infinitamente longas são estáveis. Silveira \& Flier/(2002) mostraram para o modelo de $2 \frac{1}{2}$ camadas que 0 único caso em que ondas infinitamente longas são estáveis ocorre quando $\left|h_{1} \Delta_{1}\right|=\left|h_{2} \Delta_{2}\right|$. Para 0 modelo de duas camadas, essa condição implica que $\left|\Delta_{1}\right|=\delta\left|\Delta_{2}\right|$ ou seja, a amplitude do modo barotrópico seria nula e 0 jato básico puramente baroclínico.

Os três experimentos com o modelo não-linear mostraram 0 desenvolvimento tanto do padrão de retroflexão como emissão de vórtices e dipolos vorticais pelo lóbulo de retroflexão (crista 
primária na camada superior) e no cavado primário, a partir de uma perturbação inicial imposta junto ao contorno oeste. Os cenários obtidos em muito lembram aqueles observados na Confluência Brasil-Malvinas.

Verifica-se também que os resultados do modelo não linear seguem aqueles previstos pelo modelo linear em termos de taxas de crescimento, velocidades de fase e comprimento das ondas mais instáveis, mostrando que os efeitos não lineares são responsáveis principalmente isolamento das estruturas vorticais em estruturas dipolares, que eventualmente se separam da frente e se propagam.

Duas questões podem ser levantadas em função de utilizar a dinâmica do presente modelo idealizado como 0 oceano real. A primeira é 0 fato das velocidades na camada inferior serem dominantemente no sentido oposto às de superfície. A segunda é 0 fato da necessidade de perturbação junto á margem continental. Como já mencionado no corpo do trabalho, a estrutura vertical simplicada por duas camadas requer que imponhamos um cisaIhamento bastante acentuado para substituir a complexidade do padrão de estratificação contínua do oceano real. Quanto à necessidade de imposição da perturbação, esta no oceano real pode ser induzida por variação de transporte ou da CB ou da CM. Aliás, nas simulações do modelo não lineares atribuiu-se a uma anomalia, por exemplo no transporte, ao que seria a CM idealizada.

Portanto, a utilização do modelo DC idealizado de 2 camadas indica que a instabilidade baroclínica pode ser um agente importante na formação das estruturas observadas na região da Confluência Brasil-Malvinas. Além disso o modelo de 2 camadas mostra que com apenas um modo baroclínico é possí a formação dos meandros e vórtices no modelo DC. Ao mesmo tempo 0 modo barotrópico mostra-se importante na advecção das ondas instáveis, tornando possível a formação de vórtices costeiros e padrões de retroflexão no modelo.

\section{AGRADECIMENTOS}

Cayo P.F. Francisco agradece à Fundação de Amparo à Pesquisa do Estado de São Paulo (FAPESP), Ilson C.A. da Silveira agradece à bolsa de produtividade concedida pelo Conselho Nacional de Pesquisas (CNPq). Esse trabalho é parte integrante do projeto DINBRAS-FAPESP (1998/0572-2). Também agradecemos ao Dr. Carlos Lentini pelas valorosas discussões que em muito contribuíram para esse trabalho.

\section{REFERÊNCIAS}

CAMPOS EJD \& OLSON DB. 1991. Stationary rossby waves in Western Boundary Current extensions, J. Phys. Ocean., 21: 1202-1225.

CESSI P. 1991. Laminar separation of Western Boundary Currents. J. Marine Res., 49: 697-717.

DRITSCHEL DG. 1989. Contour dynamics and contour Surgery : Numerical algorithms extended high resolution modeling of vortex dynamics in two-dimensional, inviscid, incompressible flows. Comput. Phys. Rep., 10, 79-146.

FLIERL GR. 1978. Models of vertical structure and the calibration of two layer models. Dyn. Atmos. Oceans, 2: 341-381.

GARZOLI SF \& GARRAFO Z. 1989. Transport, frontal motion and eddies at the Brazil-Malvinas Confluence. Deep Sea Res., 36(5): 681-702.

GONI GJ \& WAINER I. 2002. Investigation of the Brazil Current front variability from altimeter data. J. Geophys. Res. 106(12): 31117-21128.

GORDON AL \& GREENGROVE CL. 1986. Geostrophic circulation of the Brazil-Falkland Confluence. Deep Sea Res., Part A, 33: 573-585.

HOURY S, DOMBROWSKY E, P. De MEY \& MINSTER JF. 1987. BruntVäisällä frequency and Rossby radii in the South Atlantic. J. Phys. Ocean., 17: 1619-1626.

LENTINI CAD, OLSON DB \& PODESTÁ GP. 2002. Statistics from the Brazil Current rings observed from AVHRR: 1993 to 1998, Geophys. Res. Let., 29 (16).

LEVITUS S \& BOYER TP. 1998. World Ocean Atlas 1994, Technical Report Vol.4, National Oceanographic Data Center, Ocean Climate Laboratory, $117 \mathrm{pp}$.

NOF D \& PICHEVIN T. 1993. The Retroflection Paradox. J. Phys. Ocean., 26: 2344-2358.

OLSON D, PODESTÁ G \& BROWN 0. 1988. Temporal variations in the separation of Brazil and Malvinas currents. Deep Sea Res., 35: 19711980.

OU HW \& DE RUIJTER WPM. 1986. Separation of an inertial boundary current from a curved coastline. J. Phys. Ocean., 16: 280-289.

PEDLOSKY J. 1979. Geophysical Fluid Dynamics. 770 pp., SpringerVerlag, New York.

POLVANI LM, ZABUSKY NJ \& FLIERL GR. 1989. Two-layer geostrphic vortex dynamics. Part 1. Upper layer V-states and merger. J. Fluid Mech., 205: 215-242.

PHILLIPS NA. 1954. Energy transformations and meridional circulations associated with simple baroclinic waves in a two-level quasi-geostrophic model. Tellus, 6: 273-286. 
PULLIN DJ. 1992. Contour dynamics method. Annu. Rev. Fluid Mech., 24: 89-115.

SILVEIRA ICA da \& FLIERL GR. 2002. Eddy formation in $2 \frac{1}{2}$ layer quasigeostrophic jets. J. Phys. Oceanogr., 62: 729-745.

SILVEIRA ICA da, FLIERL GR \& BROWN WS. 1999. Dynamics of separating Western Boundary Currents. J. Phys. Oceanogr., 29: 129-144.

TOMCZAK M \& GODFREY JS. 1993. Regional Oceanography: An Introduction, 421 pp., Pergamon Press, Great Britain.

ZEMBA JC. 1991. The structure and transport of the Brazil Current between $27^{\circ} \mathrm{S}$ and $36^{\circ} \mathrm{S}$. Tese de doutoramento, Woods Hole Oceanographic Institution, Woods Hole, Massachusetts, WHOI-91-37, 160pp.

ZOU Q, OVERMAN EA, WU HM \& ZABUSKY NJ. 1988. Contour Dynamics for the Euler equations: Curvature controlled initial node displacement and accuracy. J. Comp. Phys., 78: 350-372.

\section{APÊNDICE: REVISÃO DE CALIBRAÇÃO DINÂMICA}

A técnica de calibração dinâmica, descrita por Flierl (1978), propõe a calibração do modelo de camadas, visando vincular dinamicamente o modo baroclínico do oceano real com o do oceano de duas camadas, pela obtenção da profundidade $H_{1}$ da primeira camada e do salto de densidade $\epsilon$ através de critérios quantitativos.

Flier/(1978) afirma que o comportamento do sistema aproximado é determinado por um conjunto de grandezas que são matematicamente tratadas como funcionais da estratificação do oceano real. São elas: o raio de deformação baroclínico, os valores em superfície e no fundo dos modos dinâmicos e 0 acoplamento modal.

Como o objetivo principal do presente trabalho é o estudo da confluência de correntes de contorno oeste, utilizaremos a calibração aplicada às interações não lineares. Vale ressaltar que a técnica, ao contrário do que conduzido anteriormente, é desenvolvida utilizando-se quantidades dimensionais, pois o objetivo é justamente calcular essas quantidades.

A fim de utilizarmos a calibração dinâmica é necessário conhecermos a estrutura modal da região a ser estudada. Os modos normais podem ser obtidos através da resolução da equação de vorticidade potencial quase-geostrófica. Utilizando o plano $f$, podemos escrever a Eq. 1 na sua forma dimensional e para o oceano continuamente estratificado

$\frac{\partial}{\partial t}\left(\nabla^{2}+\frac{\partial}{\partial z} \frac{f^{2}}{N^{2}} \frac{\partial}{\partial z}\right) \psi+J\left(\psi,\left[\nabla^{2}+\frac{\partial}{\partial z} \frac{f^{2}}{N^{2}} \frac{\partial}{\partial z}\right] \psi\right)=0$, onde estão presentes a variação local das vorticidade relativa e de estiramento e efeitos não lineares.
Uma possível solução para equação de vorticidade potencial, pode ser obtida utilizando-se a seguinte separação de variáveis (Flierl, 1978),

$$
\psi(x, y, z, t)=\Psi_{j}(x, y, t) F_{j}(z),
$$

tal que $\Psi_{j}$ representa a amplitude da função de corrente do jésimo modo vertical $F_{j}(z)$.

A evolução da amplitude do j-ésimo modo é dada por

$$
\frac{\partial}{\partial t}\left(\nabla^{2}-\lambda_{j}\right) \Psi_{j}+\sum_{i k} J\left(\Psi_{i}, \Psi_{k}\right) \lambda_{k} \xi_{i j k}=0 ;
$$

onde $\lambda_{j}$ é 0 autovalor associado ao j-ésimo modo vertical e

$$
\xi_{i j k}=\frac{1}{H} \int_{-H}^{0} F_{i}(z) F_{j}(z) F_{k}(z) d z
$$

é o parâmetro de acoplamento não-linear, quantizando a influência do k-ésimo modo no produto do i-ésimo e j-ésimo modos.

A estrutura vertical por sua vez é obtida pela solução do problema de Sturm-Liouville

$$
\frac{\partial}{\partial z} \frac{f^{2}}{N^{2}} \frac{\partial}{\partial z} F_{j}+\lambda_{j} F_{j}=0,
$$

ao qual são aplicadas condições de contorno de tampa rígida na superfície e fundo

$$
\frac{\partial}{\partial z} F_{j}=0 \quad z=0,-H .
$$

Os autovalores são associados ao inverso do quadrado do raio de deformação e os autovetores respeitam a condição de ortonormalidade

$$
\left(F_{i}, F_{j}\right)=\delta_{i j} .
$$

Para o modelo de camadas, os modos normais são obtidos por procedimento análogo, separando-se variáveis na forma $\psi_{i}=\alpha_{j} F_{i j}$, e $F_{i j}$ representa a amplitude do j-ésimo modo na i-ésima camada. A condição de separabilidade é agora dada na forma

$\frac{f^{2}}{\epsilon_{i-1} g H_{i}}\left(F_{j, i-1}-F_{i j}\right)-\frac{f^{2}}{\epsilon_{i} g H_{i}}\left(F_{i j}-F_{i+1, j}\right)+\lambda_{j} F_{i j}=0$, com a condição de ortonormalidade dada por

$$
\frac{1}{H} H_{i} F_{i j} F_{i k}=\delta_{j k} .
$$

Expressos em termos de quantidades dimensionais, os autovetores para o modelo de duas camadas (Eq. 5) são dados por

$$
\left[F_{01}, F_{02}\right]=[1,1] \text {, }
$$


para o modo barotrópico e

$$
\left[F_{11}, F_{12}\right]=\left[\sqrt{\frac{H_{2}}{H_{1}}},-\sqrt{\frac{H_{1}}{H_{2}}}\right],
$$

para o primeiro modo baroclínico.

Os autovetores associados, também expressos em grandezas dimensionais, são dados por

$$
\begin{aligned}
& \lambda_{0}=0, \quad R_{d 0}=\infty \\
& \lambda_{1}=\frac{f^{2}\left(H_{1}+H_{2}\right)}{\epsilon g H_{1} H_{2}} \quad, \quad R_{d 1}=\left(\frac{\epsilon g H_{1} H_{2}}{f^{2}\left(H_{1}+H_{2}\right)}\right) .
\end{aligned}
$$

A calibração para os efeitos não lineares consiste em impor que o raio de deformação baroclínico do oceano de camadas seja igual ao do oceano continuamente estratificado e que o parâmetro de acoplamento não linear associado ao primeiro modo $\xi_{111}$ do oceano de camadas seja igual ao do oceano continuamente estratificado, ou seja,

$$
\left.R_{d 1}\right|_{\text {camadas }}=\left.R_{d 1}\right|_{\text {real }} \text { e }\left.\quad \xi_{111}\right|_{\text {camadas }}=\left.\xi_{111}\right|_{\text {real }} .
$$

As quantidades $R_{d 1}, F_{i 1}$ e $\xi_{111}$ podem ser escritas em termos das expressões envolvendo $H_{1}$ e $\epsilon$, enquanto $\xi_{111}$ é dado por

$$
\left.\xi_{111}\right|_{\text {camadas }}=\frac{H_{1}}{H}\left(\frac{H_{2}}{H_{1}}\right)^{-3 / 2}-\frac{H_{2}}{H}\left(\frac{H_{2}}{H_{1}}\right)^{3 / 2} .
$$

\section{NOTAS SOBRE OS AUTORES}

Cayo Prado Fernandes Francisco é aluno do programa de Doutorado em Oceanografia Física do Instituto Oceangráfico da Universidade de São Paulo (USP). Bacharel em Física em 2001 pelo IFUSP suas áreas de interesse compreendem a dinâmica de fluidos geofísicos, instabilidades lineares e não lineares em fluidos e supercondutividade e superfluidez teóricas.

Ilson Carlos Almeida da Silveira é Doutor do Departamento de Oceanografia Física do Instituto Oceanográfico da Universidade de São Paulo (IOUSP), onde vem lecionando e desenvolvendo pesquisas desde 1997, sendo orientador de 5 doutorandos, 2 mestrandos e 2 alunos de iniciação cientíica, além de ter formado um doutor e 4 mestres. Graduado em Oceanografia pela Universidade do Estado do Rio de Janeiro (UERJ) em 1984, Mestre em Oceanografia Física pelo IOUSP em 1990 e Doutor pela University of New Hampshire em 1996, fez seu pós-doutoramento em Rhode Island no Graduate School of Oceanography (GSO), entre 1996 e 1997. Desde então, participou de vários projetos de pesquisa na qualidade de responsável e colaborador. Sua linha de pesquisa compreende a circulação de meso e larga escalas do oceano, voltada para teoria de instabilidade hidrodinâmica e para o estudo da dinâmica das correntes de contorno oeste, principalmente da Corrente do Brasil, através do desenvolvimento e utilização de modelos analíticos, semi-analíticos e numéricos. 